\title{
Histologic findings associated with laser interstitial thermotherapy for glioblastoma multiforme
}

\author{
J. Bradley Elder ${ }^{1}$, Kristin Huntoon ${ }^{{ }^{*}}$ (D), Jose Otero ${ }^{2}$, Behiye Kaya ${ }^{2}$, Jeff Hatef ${ }^{1}$, Mostafa Eltobgy ${ }^{2}$ and Russell R. Lonser ${ }^{1}$
}

\begin{abstract}
Background: Laser-interstitial thermal therapy (LITT) has been supported by some authors as an ablative treatment of glioblastoma multiforme (GBM). Although the effects of LITT have been modeled in vivo, the histologic effects in a clinical circumstance have not been described. We analyzed tissue from a patient who underwent LITT as primary treatment for GBM.

Case presentation: A 62-year-old male was diagnosed with a left temporal GBM and underwent LITT at an outside institution. Despite corticosteroid therapy, the patient was referred with increasing headache and acalculia associated with progressive peritumoral edema two weeks after LITT procedure. En bloc resection of the enhancing lesion and adjacent temporal lobe was performed with steroid-independent symptom resolution (follow-up, $>2$ years). Histologic analysis revealed three distinct histologic zones concentrically radiating from the center of the treatment site. An acellular central region of necrosis (Zone 1) was surrounded by a rim of granulation tissue with macrophages (CD68) (Zone 2; mean thickness, $1.3 \pm 0.3 \mathrm{~mm}[ \pm$ S.D.]). Viable tumor cells (identified by Ki-67, p53 and Olig2 immunohistochemistry) were found (Zone 3) immediately adjacent to granulation tissue. The histologic volume of thermal tissue ablation/granulation was consistent with preoperative (pre-resection) magnetic resonance (MR)-imaging.

Conclusion: These findings are the first in vivo in humans to reveal that LITT causes a defined pattern of tissue necrosis, concentric destruction of tumor and tissue with viable tumor cells just beyond the zones of central necrosis and granulation. Furthermore, MR-imaging appears to be an accurate surrogate of tissue/tumor ablation in the early period (2 weeks) post-LITT treatment. Surgery is an effective strategy for patients with post-LITT swelling which does not respond to steroids.
\end{abstract}

Keywords: Glioma, Glioblastoma multiforme, Histology, Laser interstitial thermotherapy, Treatment

\section{Background}

Laser-induced thermal therapy (LITT) has been used to ablate primary tumors, metastatic lesions and radiation necrosis [1-7], as well as selectively destroy epileptic foci in cases of medically-intractable epilepsy [8-11]. LITT relies on thermal tissue destruction that can be monitored by real-time magnetic resonance (MR)-imaging thermography. While the histologic effects of LITT have been described in vitro, in naïve and tumor animal

\footnotetext{
* Correspondence: Kristin.Huntoon@osumc.edu

'Department of Neurological Surgery, The Ohio State University Wexner Medical Center, 410 West 10th Avenue, Doan 1047, Columbus, OH 43210, USA

Full list of author information is available at the end of the article
}

models or inferred from MR-imaging in the clinical circumstance [3, 12-14], direct understanding of its histologic impact in human pathologic states has not been defined in humans. This information is necessary in order to fully evaluate the risk/benefits of LITT compared to classical en bloc resection of surgically accessible tumors. Insight into the effects of LITT on complex anatomic structures, its impact on neoplastic processes and clinical-imaging correlates is critical to understanding this modality.

To define the histologic and biologic effects of LITT, we systematically investigated the impact of this therapy after its use as the primary treatment of a glioblastoma multiforme (GBM). Specifically, we analyzed the

(c) The Author(s). 2019 Open Access This article is distributed under the terms of the Creative Commons Attribution 4.0 International License (http://creativecommons.org/licenses/by/4.0/), which permits unrestricted use, distribution, and 
histologic findings, effect on tumor viability surrounding treatment region and clinical-imaging correlates in a patient that underwent LITT as the initial treatment for GBM followed by resection 2 weeks later.

\section{Case presentation}

\section{Initial management}

This 62-year-old male presented to an outside institution after an acute episode of aphasia. MR-imaging was performed and revealed an enhancing left temporal mass (maximum enhancing diameter, $2.1 \mathrm{~cm}$, Fig. 1). Biopsy of the lesion was histologically consistent with GBM (IDH1R132H negative, 1p/19q intact, MGMT unmethylated, p53 positive and without EGFR amplification). The patient underwent LITT (Energy: 24.42kJ, Pulses: 876, Time: 0:29:07) of the enhancing portion of the GBM via a single treatment track approximately $4 \mathrm{~cm}$ in length. The patient was discharged home on post-treatment day 2 at his neurological baseline.

\section{Open resection}

Two weeks after LITT, despite corticosteroid therapy, the patient presented to our institution with complaints of increasing headache and calculation difficulties. MR-imaging revealed tissue necrosis with hemorrhagic material within the LITT treated region and edema surrounding the treatment site. The patient underwent en bloc resection of the enhancing lesion and adjacent tumor-infiltrated brain via a left temporal craniotomy (Fig. 2) . Patient headache and calculation difficulties resolved after surgery (discharged post-operative day 2). Post-operative MR-imaging confirmed complete resection of enhancing lesion.

\section{Clinical outcome}

He underwent adjuvant radiation therapy and was treated to a dose of 5945 cGy in 29 fractions with concurrent temozolomide. Subsequent maintenance temozolomide was discontinued after 5 cycles due to persistent pancytopenia.

Interestingly, despite no surgical or medical treatment for the past 4 years, serial MR imaging since surgery has shown no recurrence of his disease.

\section{Pathologic findings}

The gross specimen was $6.5 \mathrm{~cm}$ in the anteroposterior and $4.4 \mathrm{~cm}$ in the mediolateral dimensions. Histological examination revealed a thermal injury pattern characterized by 3 distinct staining patterns in relation to the different zones of the lesion. . First, a central necrotic zone (Zone 1) devoid of cells was present (Fig. 3a) in which there was gradual loss of staining and early resorptive changes at the margins. Surrounding the necrotic zone, an active rim (Zone 2) of granulation tissue was present (mean thickness, $1.3 \pm 0.3 \mathrm{~mm}$ ) which included vascular proliferation, lymphocytes and microglia positive for CD68 and CD45, respectively, just beyond the necrotic core and mesenchymal and glial reaction at the margin (Fig. 3b-c). Immediately beyond the granulation tissue zone, cytologically atypical, GFAP-positive astrocytes were found (Zone 3) (Fig. 3e). Immunoreactivity in these cells to OLIG2, p53, and Ki67 confirmed their neoplastic nature (Fig. 3f-h). The majority of these tumor cells showed moderately intense immunoreactivity to $\mathrm{p} 53$. IDH $1^{\mathrm{R} 132 \mathrm{H}}$ was negative in tumor cells by immunohistochemistry.

Additional immunohistochemical examination of the tissue showed hallmark features previously described in non-human tissue in vivo that has undergone LITT. In the area of viable tumor, there were noted mitoses (Fig. 4a, Zone 3). Within the transition area of from the necrotic area to the viable tumor zone was a band of granulation tissue, in which a histiocytic reaction with multinucleated giant cells was present. (Fig. 4b, Zone 2). Axonal ischemic changes such as axonal spheroids were
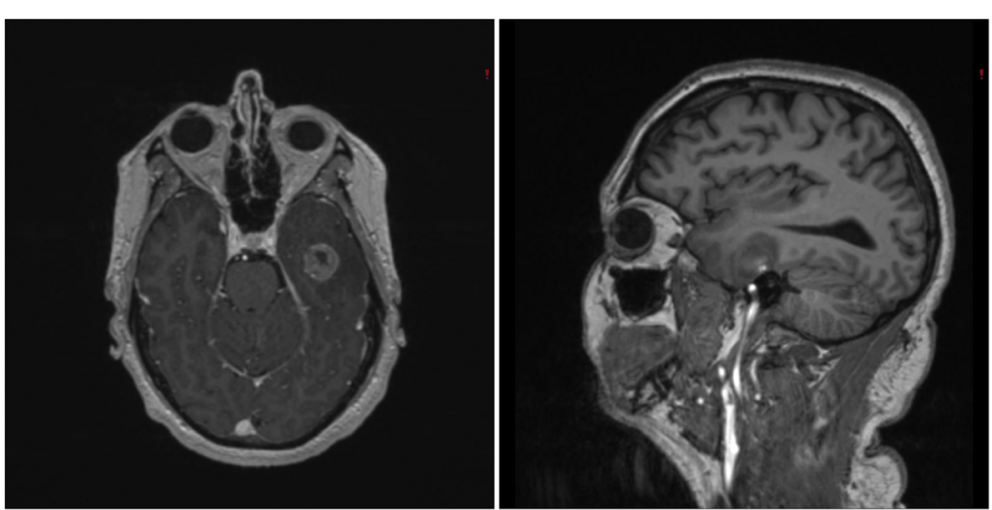

Fig. 1 Magnetic resonance (MR)-imaging at initial evaluation at outside institution. T1-weighted post-contrast MR-imaging in the axial (Left) and sagittal (Right) planes demonstrating a contrast-enhancing lesion (maximum diameter $2.1 \mathrm{~cm}$ ) that stereotactic needle biopsy confirmed to be glioblastoma multiforme. After biopsy, the patient underwent laser interstitial thermal therapy of the enhancing tumor 

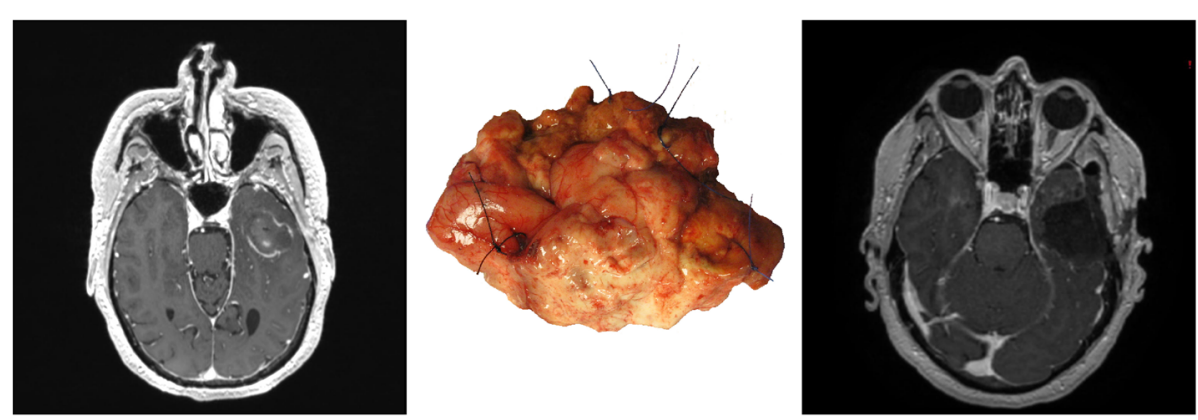

Fig. 2 Magnetic resonance (MR)-imaging and pathologic findings at resection of left temporal laser-induced thermal therapy treated glioblastoma multiforme (GBM). Preoperative (before resection) T1-weighted post-contrast MR-imaging in the axial (Left) plane demonstrates enhancing necrotic region of treatment/tumor. (Middle) En bloc resection of the enhancing GBM/treatment site and surrounding tumor-infiltrated tissue $(6.5 \mathrm{~cm}$ anteroposterior and $4.4 \mathrm{~cm}$ mediolateral dimension) was performed. Postoperative T1-weighted post-contrast MR-imaging in the axial (Right) plane demonstrates complete resection of the enhancing necrotic region of treatment/tumor

noted (Fig. 4c, Zones 1 \& 2). Moreover, cells with nuclear fragmentation indicating neuronal and cell body injury in response to laser ablation were detected (Fig. 4c, Zones 1 \& 2). Microglial cells were noted in the areas of high degrees of reactive astroglosis (Fig. 4d, Zone 2). Vascular effects of laser treatment including thrombotic occlusion of vessels were also found. These thrombotic changes were most prominent near the area of laser ablation (Fig. 4e, Zone 1).

Additionally, genomic analysis revealed the following modifications: EGFR L861Q (kinase domain) and no EGFR amplification, R108K (extracellular domain) activating mutations, PTEN G36R (missense mutation within the PTEN phosphatase domain), CDKN2A/B

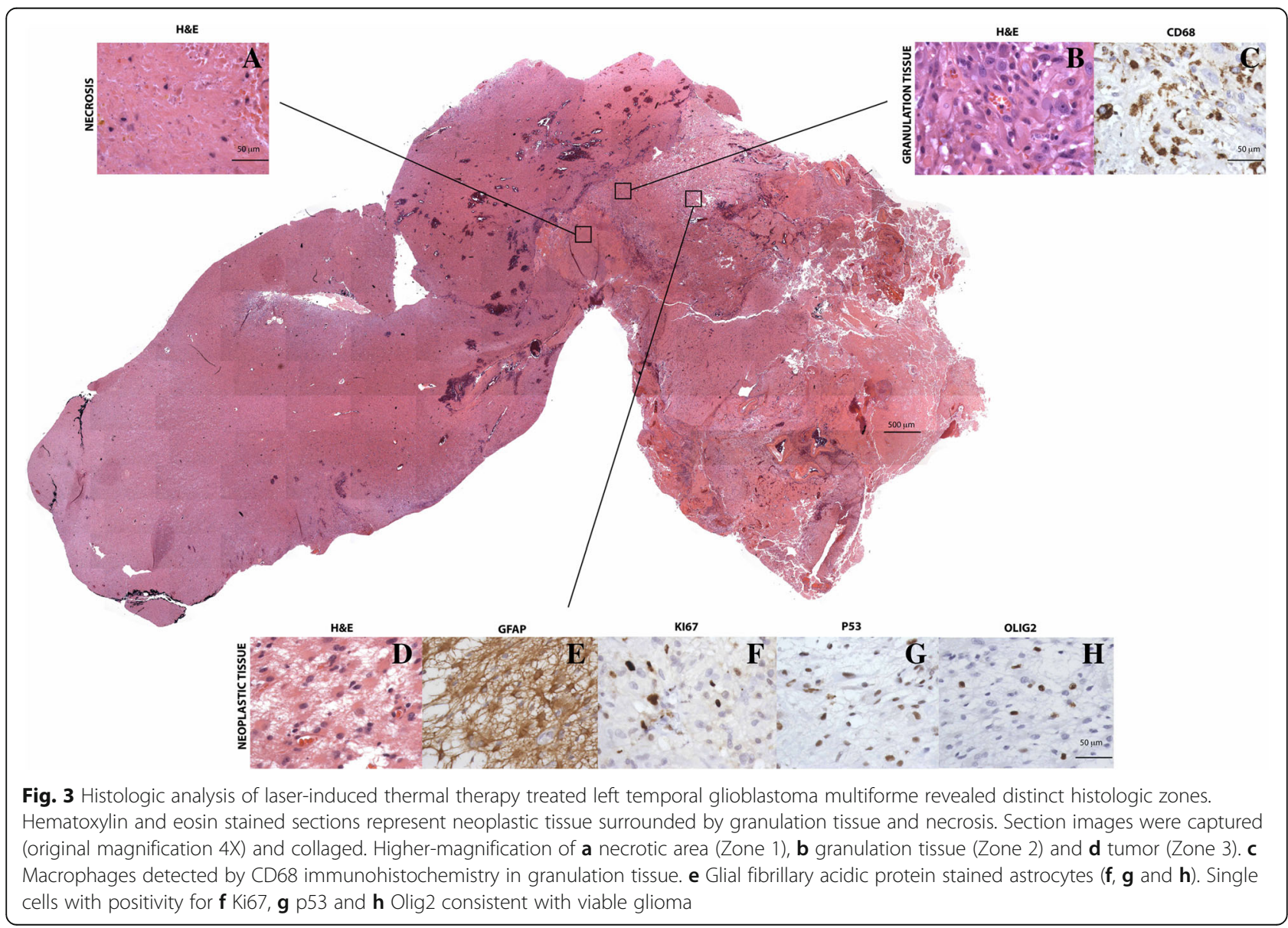




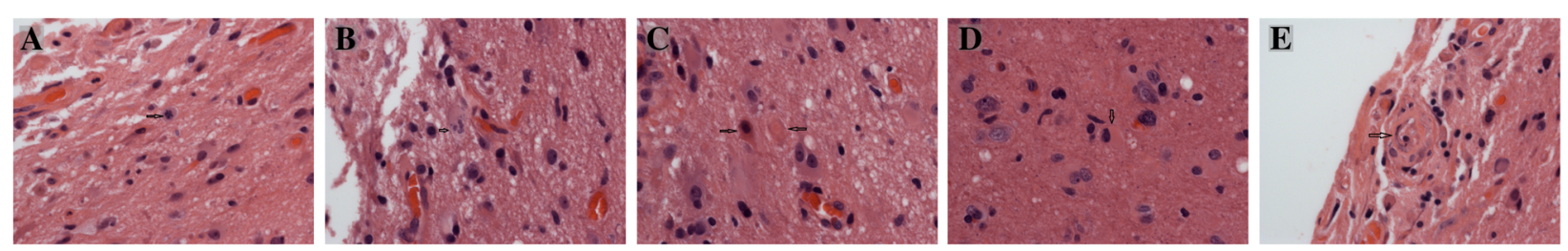

Fig. 4 Histologic analysis of laser-induced thermal therapy treated left temporal glioblastoma multiforme revealed showed some hallmark characteristics including a mitotic body (a) a multinucleated cell (b) axonal spheroid as well as an ischemic neuron (c) microglia and reactive gliosis (d) and an occluded blood vessel as well as thrombotic vascular changes (e)

loss, IDH1 negative, 1p 19q intact with a Ki-67of 25$30 \%$.

\section{Discussion}

\section{Previous findings histologic}

Prior studies examining features associated with LITT have been performed in animal models $[13,15,16]$. Specifically, defined histologic analyses in naïve animal brains have revealed that LITT creates a central region of tissue necrosis with tissue breakdown products surrounded by gliosis and edema. Histologic analysis of LITT effects in pathologic states, performed exclusively in rodent models of glioma and metastasis, has revealed that LITT ablates tumor cells/tissue resulting in similar features of central necrosis with tissue breakdown, granulation tissue formation and surrounding edema. While previous work has shown that tumor (ependymoma) control can be correlated with thermal damage on MR-imaging [7], the histologic mechanisms of tumor ablation in a patient have not been defined in humans.

To define the histologic features associated with LITT for tumor (GBM), we describe the tissue effects of this therapy in a clinical case of GBM that was not treated with other adjuvant modalities. These findings provide critical insights into the biologic, clinical and histologic features of this therapeutic modality.

\section{Current case \\ General features}

The circumstances in the current case provided a unique opportunity to better understand the histologic features of LITT for GBM. Specifically, the use of LITT as the primary treatment for GBM in this patient provided the prospect to examine the histologic features of this therapy without the confounding effects adjuvant therapy (chemotherapy, radiation or immunotherapy). The fact that the tumor was in a surgically accessible region that permitted the en bloc resection of the enhancing lesion and surrounding tumor-invaded brain tissue permitted comprehensive assessment of the pathologic/therapeutic effects of LITT in GBM. Our analysis of the histopathologic changes of the LITT area of the tumor and surrounding tumor are similar to what has been reported in mice modeling except that the degree of reactive changes surpassed what had been seen in the past. For these reasons, insights from this case can critically inform biologic and therapeutic understanding of LITT for GBM.

\section{Clinical findings}

The clinical findings in the current case reveal important characteristics associated with LITT for GBM. Previously, LITT has been associated with well-described progressive post-treatment tumor-tissue edema on MR-imaging. Imaging data indicate that the post-LITT edema develops within 3 days of treatment and can persist for several weeks (up to 6 weeks) $[12,17,18]$. Similar to other tumor-associated pathologic states [19], imaging data indicate that the post-LITT edema may be the result of treatment-related increased vascular permeability [18]. $E n$ bloc resection of the tumor as well as a region of associated edema and gliosis performed resulting in elimination of the source of edema and steroid independence.

\section{Treatment implications}

Features of the current case provide several treatment insights. First, surgical resection can be used to effectively manage post-LITT swelling refractory to corticosteroid therapy. Removal of the LITT treated volume and surrounding tissue/tumor region results in immediate improvement in tissue swelling and permits corticosteroid independence. Second, the findings support the use of MR-imaging in the early treatment period (2 weeks) to accurately define the volume of effective tumor treatment. En bloc resection of the tumor and surrounding region allowed for the precise anatomic and volumetric quantification for comparison to MR-imaging in this case. Finally, critical analysis of the effectiveness of LITT (compared to resection) and defining optimal regions for GBM treatment will be essential moving forward. Finally, this patient has survived 55 months as of this report without recurrence. If additional evidence emerges supporting a synergistic effect of LITT prior to surgical resection, then additional clinical studies may be warranted. 


\section{Histologic findings}

Similar to animal histologic studies, the current case reveals that LITT creates concentric non-specific tissue destruction extending approximately $1.5 \mathrm{~cm}$ (radius) perpendicular from the thermal source. Consistent with the tissue ablation profile, histologic analysis showed that tumor cell destruction was most effective nearest to the LITT source. Likewise, viable GBM cells were found immediately adjacent to the granulation zone surrounding the necrotic core induced by the thermal therapy. Histologic findings correlated with MR-imaging features, including areas of thermal-induced tissue destruction that appeared as a rim of contrast-enhancement surrounding a core of $\mathrm{T} 2$-weighted hyperintensity.

\section{Limitations}

The main limitation of this manuscript is the inherent limitation of being a single case reporting pathological findings that may or may not be similar to other cases of early acute post-LITT findings.

\section{Conclusion}

LITT causes a defined pattern of tissue necrosis characterized by concentric destruction of tumor and tissue, with viable tumor cells just beyond the zones of central necrosis and granulation. MR-imaging is an accurate surrogate of tissue/tumor ablation in the early period ( 2 weeks) after treatment. Swelling caused by LITT can be effectively treated with surgical resection of the lesion. Further histologic studies are needed to more fully characterize the immediate, intermediate and long-term effects of LITT on various intracranial pathologies.

\section{Abbreviations}

GBM: Glioblastoma multiforme; LITT: Laser-interstitial thermal therapy

\section{Acknowledgements}

We appreciate the contributions of the patient and his family in allowing us to discuss his care and findings.

\section{Funding}

There was no funding source for this manuscript.

\section{Availability of data and materials}

The data used and/or analyzed during the current study are available from the corresponding author on reasonable request.

\section{Authors' contributions}

JE was a major contributor in writing the manuscript and concept of the manuscript; $\mathrm{KH}$ was a major contributor in writing the manuscript and compiling figures; JO was a contributor to the pathological analysis; BK compiled figures and pathological analysis; $\mathrm{JH}$ helped in writing the manuscript; ME compiled figures; and RL was a major contributor in writing the manuscript. All authors read and approved the final manuscript.

\section{Ethics approval and consent to participate}

All of the intended procedures in the present study, including the use of specimens from human subjects, were approved with the written informed consent of the patient and next of kin, according to the guidelines of the Ohio State University. Ethical approval was not obtained nor required for this case presentation.

\section{Consent for publication}

Written informed consent was obtained from the patient for the publication of this case report and any accompanying images. A copy of the consent form is available for review by the Editor of this journal.

\section{Competing interests}

The authors declare that they have no competing interests.

\section{Publisher's Note}

Springer Nature remains neutral with regard to jurisdictional claims in published maps and institutional affiliations.

\section{Author details}

'Department of Neurological Surgery, The Ohio State University Wexner Medical Center, 410 West 10th Avenue, Doan 1047, Columbus, OH 43210,

USA. ${ }^{2}$ Division of Neuropathology, Department of Pathology, The Ohio State University Wexner Medical Center, Columbus, OH, USA.

Received: 30 August 2018 Accepted: 1 February 2019

Published online: 15 February 2019

\section{References}

1. Banerjee C, Snelling B, Berger MH, Shah A, Ivan ME, Komotar RJ. The role of magnetic resonance-guided laser ablation in neurooncology. $\mathrm{Br} J$ Neurosurg. Apr 2015;29(2):192-6.

2. Rao MS, Hargreaves EL, Khan AJ, Haffty BG, Danish SF. Magnetic resonanceguided laser ablation improves local control for postradiosurgery recurrence and/or radiation necrosis. Neurosurgery Jun 2014;74(6):658-667; discussion 667.

3. Reimer P, Bremer C, Horch C, Morgenroth C, Allkemper T, Schuierer G. MRmonitored LITT as a palliative concept in patients with high grade gliomas: preliminary clinical experience. J Magn Reson Imaging. Jan-Feb 1998;8(1): 240-4.

4. Jethwa PR, Barrese JC, Gowda A, Shetty A, Danish SF. Magnetic resonance thermometry-guided laser-induced thermal therapy for intracranial neoplasms: initial experience. Neurosurgery Sep 2012;71(1 Suppl Operative): 133-144; 144-135.

5. Jethwa PR, Lee JH, Assina R, Keller IA, Danish SF. Treatment of a supratentorial primitive neuroectodermal tumor using magnetic resonanceguided laser-induced thermal therapy. J Neurosurg Pediatr Nov. 2011;8(5): 468-75.

6. Mohammadi AM, Hawasli AH, Rodriguez A, et al. The role of laser interstitial thermal therapy in enhancing progression-free survival of difficult-to-access high-grade gliomas: a multicenter study. Cancer Med. 2014;3(4):971-9.

7. Patel NV, Jethwa PR, Shetty A, Danish SF. Does the real-time thermal damage estimate allow for estimation of tumor control after MRI-guided laser-induced thermal therapy? Initial experience with recurrent intracranial ependymomas. J Neurosurg Pediatr Apr. 2015;15(4):363-71.

8. Hawasli AH, Bandt SK, Hogan RE, Werner N, Leuthardt EC. Laser ablation as treatment strategy for medically refractory dominant insular epilepsy: therapeutic and functional considerations. Stereotact Funct Neurosurg. 2014;92(6):397-404.

9. Kang JY, Wu C, Tracy J, et al. Laser interstitial thermal therapy for medically intractable mesial temporal lobe epilepsy. Epilepsia. Feb 2016;57(2):325-34.

10. Gross RE, Willie JT, Drane DL. The role of stereotactic laser Amygdalohippocampotomy in mesial temporal lobe epilepsy. Neurosurg Clin N Am Jan. 2016;27(1):37-50.

11. McCracken DJ, Willie JT, Fernald BA, et al. Magnetic resonance thermometry-guided stereotactic laser ablation of cavernous malformations in drug-resistant epilepsy: imaging and clinical results. Neurosurgery. 2015: 25.

12. Schwabe B, Kahn T, Harth T, Ulrich F, Schwarzmaier HJ. Laser-induced thermal lesions in the human brain: short- and long-term appearance on MRI. J Comput Assist Tomogr. 1997;21(5):818-25.

13. Schulze PC, Kahn T, Harth T, Schwurzmaier HJ, Schober R. Correlation of neuropathologic findings and phase-based MRI temperature maps in experimental laser-induced interstitial thermotherapy. J Magn Reson Imaging. 1998:8(1):115-20.

14. Tracz RA, Wyman DR, Little PB, et al. Comparison of magnetic resonance images and the histopathological findings of lesions induced by interstitial laser photocoagulation in the brain. Lasers Surg Med. 1993;13(1):45-54. 
15. Schulze PC, Adams V, Busert C, Bettag M, Kahn T, Schober R. Effects of laserinduced thermotherapy (LIT) on proliferation and apoptosis of glioma cells in rat brain transplantation tumors. Lasers Surg Med. 2002;30(3):227-32.

16. Schulze PC, Vitzthum HE, Goldammer A, Schneider JP, Schober R. Laserinduced thermotherapy of neoplastic lesions in the brain--underlying tissue alterations, MRI-monitoring and clinical applicability. Acta Neurochir. 2004; 146(8):803-12.

17. Tiwari P, Danish S, Madabhushi A. Identifying MRI markers associated with early response following laser ablation for neurological disorders: preliminary findings. PLoS One. 2014;9(12):e114293.

18. Leuthardt EC, Duan C, Kim MJ, et al. Hyperthermic laser ablation of recurrent glioblastoma leads to temporary disruption of the peritumoral blood brain barrier. PLoS One. 2016;11(2):e0148613.

19. Lonser RR, Vortmeyer AO, Butman JA, et al. Edema is a precursor to central nervous system peritumoral cyst formation. Ann Neurol. 2005;58(3):392-9.

Ready to submit your research? Choose BMC and benefit from:

- fast, convenient online submission

- thorough peer review by experienced researchers in your field

- rapid publication on acceptance

- support for research data, including large and complex data types

- gold Open Access which fosters wider collaboration and increased citations

- maximum visibility for your research: over $100 \mathrm{M}$ website views per year

At $\mathrm{BMC}$, research is always in progress.

Learn more biomedcentral.com/submissions 